\title{
Feedback stabilization of non-homogeneous bilinear systems with a finite time delay
}

\author{
Zakaria Hamidi ${ }^{1}$, Rachid El Ayadi ${ }^{2}$, and Mohamed Ouzahra ${ }^{3}$ \\ ${ }^{1}$ Universite Sidi Mohamed Ben Abdallah \\ ${ }^{2}$ Sidi Mohamed Ben Abdellah University Faculty of Science and Technology of Fez \\ ${ }^{3}$ Sidi Mohamed Ben Abdellah University
}

June 15, 2021

\begin{abstract}
This paper investigates the feedback stabilization of non-homogeneous delayed bilinear systems, evolving in Hilbert state space. More precisely, under observability like assumption, we prove the exponential and strong stability of the solution by using a bounded feedback control. The partial stabilization is discussed as well. The proof of the main results is based on the decomposition method. The decay estimates of the corresponding solution are obtained. Finally, some examples are presented.
\end{abstract}

\section{Hosted file}

HAMIDI ET AL. Finale Version .pdf available at https://authorea.com/users/419973/articles/ 526336-feedback-stabilization-of-non-homogeneous-bilinear-systems-with-a-finite-timedelay 